\title{
IP-in-IP Tunneling to Enable the Simultaneous Use of Multiple IP Interfaces for Network Level Connection Striping
}

\author{
Dhananjay S. Phatak Tom Goff Jim Plusquellic \\ Department of Computer Science and Electrical Engineering \\ University of Maryland Baltimore County (UMBC) \\ 1000 Hilltop Circle \\ Baltimore, MD 21250 \\ Email: \{phatak, tgoff1, plusquel\}@umbc.edu
}

March 14, 2003

\begin{abstract}
With ubiquitous computing and network access now a reality, multiple network conduits are become widely available to mobile as well as static hosts: for instance wired connections, 802.11 style LANs, Bluetooth, and cellular phone modems. Selection of the preferred mode of data transfer is a dynamic optimization problem which depends on the type of application, its bandwidth/latency/jitter requirements, current network conditions (such as congestion or traffic patterns), cost, power consumption, battery life, and so on. Furthermore, since wireless bandwidth is likely to remain a scarce resource, we foresee scenarios wherein mobile hosts will require simultaneous data transfer across multiple IP interfaces to obtain higher overall bandwidth.

We present a brief overview of existing work which enables the simultaneous use of multiple network interfaces and identify the applicability as well as strengths and weaknesses of these related approaches. We then propose a new mechanism to aggregate the bandwidth of multiple IP paths by splitting a data flow across multiple network interfaces at the IP level. We have analyzed the performance characteristics of our aggregation scheme and demonstrate significant gains when the network paths being aggregated have similar bandwidth and latency characteristics. In addition, our method is transparent to transport (TCP/UDP) and higher layers, and allows the use of multiple network interfaces to enhance reliability. Our analysis identifies the conditions under which the proposed scheme, or any other scheme that stripes a single TCP connection across multiple IP paths, can be used to increase throughput.
\end{abstract}

\section{Introduction}

As wireless networks, services, and computing continue their explosive growth it is clear that multiple network transport mechanisms will become available to hosts. For instance, a mobile host might have

This work was supported in part by Aether Systems Inc. and NSF grants ECS-9875705 and ECS-0196362.

Portions of this work appeared in: D. S. Phatak and T. Goff, "A Novel Mechanism for Data Streaming Across Multiple IP Links for Improving Throughput and Reliability in Mobile Environments,” in IEEE INFOCOM, pp. 773-781, June 2002. 
access to the Internet via multiple networking technologies such as Bluetooth, wireless LANs (802.11, Airport LANs, Ricochet, etc.), and cellular phone modem where each technology has a corresponding service provider. Selecting which service to use for data transfer is a dynamic optimization problem which should track time varying attributes which might include bandwidth/throughput, latency, jitter, quality of service (QoS) requirements, cost, power consumption, residual battery life, interference, and traffi c patterns. Also, wireless bandwidth is a scarce resource which is unlikely to improve at the same pace as the rapid growth in bandwidth available via wired networks. Hence it is probable that mobile users will want to simultaneously stream data across multiple network interfaces in order to best make use of all available bandwidth. Therefore, this paper investigates the simultaneous use of multiple network interfaces to enhance the overall bandwidth available to a wireless node. An immediate secondary advantage is increased reliability, for example if one network interface goes down or one network path becomes severely congested the end-to-end connection is not interrupted.

In some cases, such as military applications, multiple identical or very similar network access mechanisms are provided for the sake of reliability in hostile environments. Although though the primary motivation for providing redundant network access is reliability, it would be desirable to stream distinct data packets across all interfaces simultaneously whenever possible. This would be a "performance enhancement" mode. When reliability becomes an issue, the data transfer could switch to a "reliability fi rst" mode, where two or more identical copies of the data stream might be sent across multiple access points in the hope that at least one reaches its destination. Ideally there would be no hard cutoff between the performance enhancement and reliability fi rst modes. In general a mixture could exist, for example only duplicate lost packets for transfer across multiple interfaces. Furthermore, the transition between performance-enhancement and reliability fi rst modes should happen dynamically in reaction to the environment.

\subsection{Problem Definition}

Figure 1 illustrates the case when hosts $A$ and $B$ want to communicate via the Internet and both have multiple transport conduits. For instance, host $A$ might be a laptop at an airport with access to the Internet via an 802.11 LAN (say interface $A_{1}$ ), a Bluetooth scatternet $\left(A_{2}\right)$, and a cellular phone modem $\left(A_{3}\right)$. In general, the IP addresses assigned to interfaces $A_{1}, A_{2}$, and $A_{3}$ will be controlled by separate Internet Service Providers (ISPs) who might in turn implement fi rewalling to various degrees.

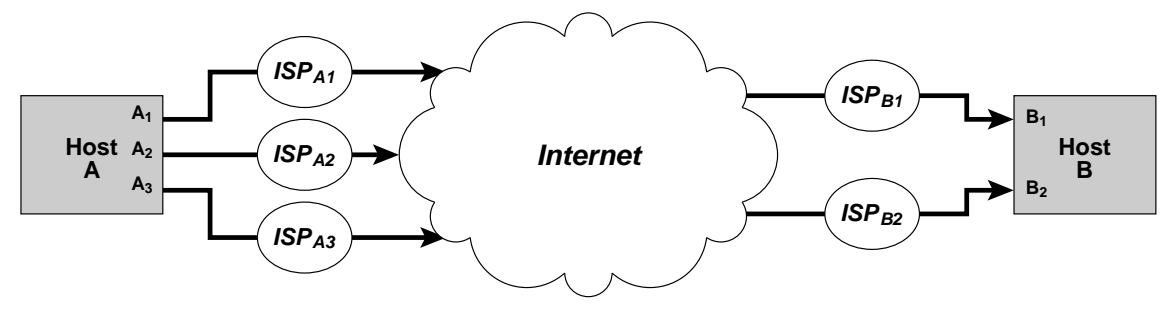

Figure 1: Communicating Hosts With Multiple Network Connections (Multi-Homed)

Suppose $A$ wants to stream data to $B$ across multiple interfaces to enhance the overall bandwidth. Several questions arise when considering the possible ways to achieve this effect. Should the data stream be split into multiple streams at the application level? In this case the application would open multiple connections across different network interfaces and be responsible for splitting the data stream at the server and 
merging it properly at the client. This approach might be too cumbersome and restrictive since the number of connections might have to be decided in advance in order to fi gure out how many flows to split the traffi c into. Alternatively, should the splitting be done at transport layer or at the network layer? In essence we are considering this general question of striping a connection across multiple IP interfaces for bandwidth aggregation.

\subsection{Related Work}

Bandwidth aggregation across multiple channels has been considered in the literature in varying contexts and for different applications and scenarios, distinct from those considered in this paper. For instance, multilink PPP [1, 2, 3] is designed to aggregate multiple logical data channels into one. Since PPP was intended to operate at the data link layer, below the IP level [4, 5, 6], the multi-link PPP extension bundles multiple data-link level channels into a single logical link. In the scenario we are considering, links have different IP addresses assigned and controlled by completely independent Internet Service Providers (ISPs). It is highly unlikely that independent ISPs will allow arbitrary users to bundle their links into "one logical link". Thus, PPP would have to run on-top-of the IP layer which is not its intended use, and has its own set of diffi culties.

Multi-path and QoS routing have been considered in wired as well as wireless networks, a sampling can be found in $[7,8,9,10,11,12,13,14,15,16]$. Multi-path routing in wired networks concentrates mainly on the network layer. The issue of splitting a single connection into multiple streams is not addressed. Multipath and QoS routing work in mobile ad hoc networks also focuses on routing at the network layer. Here the assumption is that there is a single radio interface which is used to fi nd distinct routes to a destination via multiple neighbors within listening range of the source. This is different from considering multiple interfaces and striping the same connection across those interfaces. We would like to point out that the issues addressed in multi-path QoS routing are relevant to the problem at hand: it would be good to use disjoint shortest path pairs, incorporate QoS attributes, etc. Fundamentally, however, these issues are different from the problem of effi ciently striping a single connection across multiple IP paths. Thus the multi-path QoS routing results found in the literature can be used in conjunction with a connection striping scheme.

Bandwidth aggregation has also been considered in the context of storage systems and high volume data or fi le servers, for instance [17, 18, 19, 20]. Typical storage-server architectures are confi ned to within a LAN having a single controlling authority. Note that within a LAN it is possible to do bandwidth aggregation at the MAC layer. For instance, Cisco's EtherChannel [21] product provides bandwidth aggregation across multiple Ethernet links. This product targets the extremely high instantaneous bandwidth requirements between a data-server and nodes which services queries in typical database applications. Likewise, a recent IETF draft from the HP storage systems group [20] deals with exploiting ultra wide-band SCSI connections for distributed storage. They simply mention the problems associated with trying to aggregate multiple IP paths into a single TCP connection; they don't propose a solution.

The recently proposed "Stream Control Transmission Protocol" (SCTP, RFC 2960, and [22, 23, 24, 25, 26]) comes closest to solving the problem at hand. SCTP is a new transport level protocol which supports multi-streaming and multi-homing (a host having multiple IP addresses). A recently proposed extension [27] would allow the dynamic addition/deletion of IP addresses at both the source and destination. However, in its current form SCTP does not perform load-sharing, that is multi-homing is used only for redundancy [25] as follows. A single IP address is chosen as the primary address for a connection, meaning the destination to which all normally transmitted data chunks are sent. Retransmitted data chunks may use an alternate 
destination address to improve the probability of reaching a remote endpoint. Persistent send failures to a primary address will ultimately result in choosing a new primary address for the connection.

To the best of our knowledge bandwidth aggregation across multiple IP paths has not been considered in the context of mobile/wireless networks where we believe it will be a real issue. In the following sections we propose a new mechanism for bandwidth aggregation across multiple IP paths and analyze its performance.

\section{Proposed Aggregation Mechanism}

We propose a bandwidth aggregation solution at the network (IP) layer. The idea is to manipulate the routing entities (daemons, tables, etc.) so as to send packets through different network interfaces. This approach has the following advantages.

(i) To transport, application, and higher layers the data stream looks like a single flow. Therefore all existing applications can benefi $\mathrm{t}$ transparently, without being rewritten to explicitly do stream-splitting themselves.

(ii) This approach is independent of underlying network technologies, e.g. 802.11, Bluetooth. The prevalence of TCP/IP makes IP support likely for most common network technologies. Note that even if the actual network layer protocol is not IP, we propose implementing aggregation at the network level.

(iii) The proposed approach can be highly dynamic. If the route through an interface becomes congested packets can simply be sent through an alternate interface. In the discussion that follows we show some problems that can arise from this approach.

(iv) Reliability can be enhanced. If one interface goes down, another interface can be used.

(v) Likewise, once such a mechanism is available it could be employed to optimize parameters other than reliability, such as power consumption, latency, jitter, etc.

Since TCP is used more predominantly than UDP from inter-LAN communication, we illustrate our method for TCP (although it can also be used for UDP flows). For the purpose of illustration, assume that node $A$ is the source and node $B$ is the destination, as shown in Figure 1. The IP address associated with interface $A_{i}$ is denoted $\operatorname{Addr}\left(A_{i}\right)$, and the IP address associated with interface $B_{i}$ is denoted $\operatorname{Addr}\left(B_{i}\right)$. Assume that $A$ only knows how to reach $B$ using the IP address associated with interface $B_{1}$. For now, also assume that $A$ will only send data to $B$ using interface $B_{1}$, the more general case where both $A$ and $B$ use multiple interfaces will be considered later.

For the scenario described above, the initial SYN packet for the TCP connection from $A$ to $B$ goes through interfaces $A_{1}$ and $B_{1}$. The TCP stack at host $B$ logs the source address of the connection as being associated with interface $A_{1}$, i.e. $\operatorname{Addr}\left(A_{1}\right)$. Likewise the connection identifi er at $A$ has $\operatorname{Addr}\left(B_{1}\right)$ as the destination address for the connection. Now suppose the application at $A$ requires more bandwidth and packets from the single transport layer connection with $B$ are simultaneously routed through an alternate network interface, say interface $A_{2}$. This leads to the following problems.

(i) Typically, the source address of a packet is the address associated with the interface through which it is sent out. Therefore, packets from a connection that was established with source address $\operatorname{Addr}\left(A_{1}\right)$ will 
contain the unrecognized source address $\operatorname{Addr}\left(A_{2}\right)$ when sent through $A_{2}$. When the packet reaches its destination, $B$, it is not recognized as belonging to an established connection since a TCP connection is uniquely identifi ed by the tuple (source IP address, source port, destination IP address, destination port). The packet is then dropped by $B$.

(ii) Alternatively, suppose the source address $\operatorname{Addr}\left(A_{1}\right)$ is assigned to packets sent out through $A_{2}$. As a side effect, these packets now appear spoofed to intermediate routers. For security reasons spoofed packets are almost certainly dropped by ISPs. Therefore packets sent through $A_{2}$ still do not reach the destination application at $B$.

Our solution to these problems is to employ tunneling. At the sender side, $A$, the transport layer assembles all packets as if they were going through $A_{1}$ and addressed to $B_{1}$. The packets going out on interface $A_{2}$ then get encapsulated in a new IP packet with an extra header having destination address $B_{1}$ and source address $A_{2}$. The protocol fi eld for the outer header should be IP-in-IP (also used for tunneling in the mobile IP standard and implementations [28, 29, 30, 31]). The destination, $B$, can then recognize IP-in-IP packets and strip the outer header. This leaves the original packet with proper source and destination addresses to be delivered up the network stack to TCP in a transparent manner. The TCP stack at $B$ is unaware that $A$ tunneled the packet through an alternate interface.

Note that the same tunneling mechanism can be used when $B$ is accepting data on multiple interfaces. For instance, to send a packet through interfaces $A_{3}$ and $B_{2}$, the original packet (having source and destination addresses $\operatorname{Addr}\left(A_{1}\right)$ and $\operatorname{Addr}\left(B_{1}\right)$ respectively) is encapsulated in a packet having source address $\operatorname{Addr}\left(A_{3}\right)$ and destination address $\operatorname{Addr}\left(B_{2}\right)$. The receiving IP stack at $B$ strips the outer header, realizes that the inside packet is for an existing TCP connection, and transparently passes it to TCP.

While this scheme presents a feasible solution, it raises the following issues.

(i) How does a source learn multiple IP addresses for a destination and vice versa?

(ii) Many radio technologies operate in the same frequency band, for example Bluetooth and 802.11. Using Bluetooth and 802.11 simultaneously might lead to interference between the two, causing degraded performance.

(iii) The IP-in-IP encapsulation adds some processing overhead.

(iv) TCP performance could be adversely effected. TCP was designed to work well across a single network path not multiple paths. If the bandwidth/delay characteristics of two paths are substantially different, packets sent on the lower bandwidth path will cause timeouts and/or fast retransmits. This will cause the sender to throttle its transmission rate via congestion window reduction, slow start, and other congestion avoidance mechanisms employed by TCP. The net effect could be that the lower bandwidth path "drags down" the higher bandwidth path. This would defeat the original purpose of using multiple interfaces simultaneously, in which case using only the higher bandwidth path would be optimal.

We address each of the above issues. The fi rst three are discussed in the remainder of this section while the last one is considered in the following section.

The fi rst issue is how to make $A$ and $B$ aware of any multiple IP addresses the other may have. To accomplish this, IP and/or TCP headers and their processing would have to be modifi ed. This is similar to 
the solution in SCTP which allows connection initialization chunks to list multiple addresses. While such modifi cations might interfere with interoperability, we believe that using some more of the full header length allowed (by TCP, IPv4 and IPv6) or employing some of the unused fi elds is a fairly transparent solution.

The second question, as to whether using multiple interfaces is tantamount to creating self interference, for example when the same wireless spectrum is used, is beyond the scope of this work and highly technology specifi c. For example, while Bluetooth and 802.11 occupy the same band, while cellular phone modems and the projected wideband CDMA do not. In these cases the radio interference issue is nonexistent. With the rapid growth in wireless technologies and services, the chances are small that all transport conduits will end up using same technology and/or spectrum. Also, since our solution is at the IP level it can be applied to wired scenarios as well. A typical desktop at a campus today may likely have a wired Ethernet connection as well as an 802.11 wireless LAN connection, where the wireless cell data rate can be $11 \mathrm{Mbps}$ or higher. The proposed connection striping scheme could be applied in this case, with some packets being sent across the wired interface and some across the wireless link. In this case there is obviously no interference between the two interfaces.

The third issue deals with the IP-in-IP overhead. Experimental data shows that this overhead is negligible (see Section 5). Note that packets sent on the primary path, i.e. the path over which the TCP SYN packet was sent that established the source and destination addresses, need not be tunneled and do not incur the IP-in-IP encapsulation overhead. Assuming that the primary path is the highest bandwidth path, most of the packets are then free from the IP-in-IP overhead. Furthermore if tunneling is not done, the lower bandwidth path(s) are not used at all. By employing the proposed scheme, one can hope to utilize at least some part of the spare capacity of the lower bandwidth path(s). Thus any added bandwidth utilization is available for free, meaning it is simply not realizable by the conventional single path TCP connection, in this sense the term "overhead" is a misnomer. In addition, the minimal encapsulation proposed in the mobile IP standard [30] along with header compression techniques can be employed as well to mitigate any IP-in-IP overhead.

Now only the last question from above remains, the question of TCP performance. This is considered in detail in Section 4, however fi rst a quantitative analysis of data splitting is presented.

\section{Splitting a Data Stream}

Splitting a single transport layer (TCP) stream into multiple network layer (IP) streams can be accomplished in many ways. In this section we present an analytical model of a network path and fi nd how data show per divided between paths to best make use of the available network resources by maximizing overall throughput. However, an exact packet scheduling algorithm is not considered here. Many alternate approaches might be appropriate, each having tradeoffs, the study of which is beyond the current scope and a matter for future work.

\subsection{Analytical Path Model}

Consider two network nodes, node $A$ and node $B$, where node $A$ is sending data to node $B$. Assume there are $N$ distinct paths from $A$ to $B$, where the time needed to send a packet along path $k$ is a linear function of packet size. That is, the one-way latency of the $i$-th packet of size $p_{i}$, sent from $A$ to $B$ along path $k$ at time $t$ 
is

$$
l_{A B_{k}}\left(p_{i}, t\right)=\frac{p_{i}}{b_{A B_{k}}(t)}+c_{A B_{k}}(t) .
$$

Given this model, $b_{A B_{k}}(t)$ and $c_{A B_{k}}(t)$ correspond to the effective bandwidth and propagation delay, respectively, of the $k$-th path from $A$ to $B$ at time $t$. From this one-way latency, the RTT of the $i$-th packet becomes

$$
r\left(p_{i}, q_{i}, t\right)=l_{A B_{k}}\left(p_{i}, t\right)+\delta_{i}+l_{B A_{k^{\prime}}}\left(q_{i}, t+l_{A B_{k}}\left(p_{i}, t\right)+\delta_{i}\right),
$$

where $\delta_{i}$ is the delay between when packet $i$ was received and when the corresponding ACK was sent, and $q_{i}$ is the size of the packet sent from $B$ to $A$ containing the ACK.

To make the ensuing analysis more manageable, the following simplifying assumptions are made.

- The effective bandwidth of a path is constant throughout the lifetime of a TCP connection, this allows the time dependency to be dropped from (1) and (2).

- All data packets sent along path $k$ have same size $p_{k}$.

- There is no delay between when a packet is received and when the corresponding ACK is sent, that is $\delta_{i}=0$ in (2).

- All ACKs are sent back on a single path on which the TCP SYN packet was sent. We also assume that ACK packets are much smaller than data packets. Since all ACKs are sent back on the same path, the latency of transmission for all ACKs can be assumed to be the same: $\Delta_{\text {ack }}$.

These assumptions simplify (2), and the RTT of a packet sent along path $k$ becomes

$$
r_{k}=l_{k}\left(p_{k}\right)+\Delta_{\mathrm{ack}}=\frac{p_{k}}{b_{k}}+c_{k}+\Delta_{\mathrm{ack}}
$$

\subsection{Optimal Portion of Data Sent on Each Path}

To fully utilize the available network resources, data should be sent on all paths between $A$ and $B$ throughout the life of a TCP connection. In other words, the time needed to send $n_{k}$ packets each of size $p_{k}$ along path $k$ should equal the total connection time, leaving no path idle during the connection. This means

$$
L_{1}\left(p_{1}, n_{1}\right)=L_{2}\left(p_{2}, n_{2}\right)=\cdots=L_{N}\left(p_{N}, n_{N}\right),
$$

where $L_{i}\left(p_{i}, n_{i}\right)$ is the total transfer time for data sent on path $i$. Assuming packets are sent in a pipelined fashion, where multiple packets are allowed in flight simultaneously, this total transfer time becomes

$$
L_{i}\left(p_{i}, n_{i}\right) \approx \frac{p_{i} \cdot n_{i}}{b_{i}}+c_{i}
$$

The system of equations given in (4) then becomes

$$
\frac{n_{1} \cdot p_{1}}{b_{1}}+c_{1}=\frac{n_{2} \cdot p_{2}}{b_{2}}+c_{2}=\cdots=\frac{n_{N} \cdot p_{N}}{b_{N}}+c_{N} .
$$


Alternatively, this can be expressed in terms of the total amount of data having size $D$ to be transferred from $A$ to $B$, where $D=\sum_{i} n_{i} p_{i}$. Noting that the fraction of total data sent along path $i$ is

$$
f_{i}=\frac{n_{i} \cdot p_{i}}{D},
$$

equation (6) becomes:

$$
\frac{D}{b_{1}} \cdot f_{1}+c_{1}=\frac{D}{b_{2}} \cdot f_{2}+c_{2}=\cdots=\frac{D}{b_{N}} \cdot f_{N}+c_{N}
$$

where

$$
f_{1}+f_{2}+\cdots+f_{N}=1
$$

This system of equations defi ned by (8) and (9) can be represented in matrix form as

$$
\mathbf{B f}=\mathbf{c} .
$$

Here $\mathbf{B}$ is an $N \times N$ matrix whose elements are defi ned by

$$
\begin{array}{ll}
\mathbf{B}_{i j}= \begin{cases}b_{i}^{-1} & \text { if } j=i \\
-b_{i+1}^{-1} & \text { if } j=i+1 \\
0 & \text { otherwise }\end{cases} & \text { For rows } i<N \\
\mathbf{B}_{i j}=1 & \text { For row } i=N,
\end{array}
$$

$\mathbf{f}$ is a column vector whose elements are the fraction of data sent along the corresponding path, or $\mathbf{f}_{i}=f_{i}$, and $\mathbf{c}$ is a column vector where

$$
\mathbf{c}_{i}=\left\{\begin{array}{ll}
\frac{c_{i+1}-c_{i}}{D} & \text { if } i<N \\
1 & \text { if } i=N
\end{array} .\right.
$$

This makes (10)

$$
\underbrace{\left[\begin{array}{ccccc}
b_{1}^{-1} & -b_{2}^{-1} & & & \\
& b_{2}^{-1} & -b_{3}^{-1} & & \\
& & \ddots & \ddots & \\
& & & b_{N-1}^{-1} & -b_{N}^{-1} \\
1 & \cdots & \cdots & \cdots & 1
\end{array}\right]}_{\mathbf{B}} \underbrace{\left[\begin{array}{c}
f_{1} \\
f_{2} \\
\vdots \\
f_{N-1} \\
f_{N}
\end{array}\right]}_{\mathbf{f}}=\underbrace{\left[\begin{array}{c}
\frac{c_{2}-c_{1}}{D} \\
\frac{c_{3}-c_{2}}{D} \\
\vdots \\
\frac{c_{N}-c_{N-1}}{D} \\
1
\end{array}\right]}_{\mathbf{c}} .
$$

Due to its construction, this system of equations is guaranteed to have a unique solution, given by

$$
\mathbf{f}=\mathbf{B}^{-1} \mathbf{c}
$$

For large $D$, the terms $\frac{c_{i}-c_{i+1}}{D}$ approach zero, leading to the solution

$$
f_{i}=\frac{b_{i}}{b_{1}+b_{2}+\cdots+b_{N}} .
$$

In other words, the fraction of data sent along a given path corresponds to the ratio of its bandwidth to the total available bandwidth. This can be verifi ed intuitively as follows. 
For large $D$ the transfer time, $t$, is going to be bandwidth dominated, under the pipelined transfer assumption, where

$$
t=\frac{D}{b_{\text {total }}} \quad \text { and } \quad b_{\text {total }}=b_{1}+b_{2}+\cdots+b_{N} .
$$

In this time, the fraction of data sent along path $i, D_{i}$, using the full bandwidth of the path is

$$
D_{i}=b_{i} \cdot t=\frac{b_{i} \cdot D}{b_{\text {total }}} .
$$

The fraction of total data sent along path $i$ is then

$$
f_{i}=\frac{D_{i}}{D}=\frac{b_{i}}{b_{\text {total }}} .
$$

Therefore, for large data transfers, data should be partitioned across multiple paths according to (14) in order to minimize the overall transfer time.

\section{TCP Performance Analysis}

At the outset, we would like to point out that the proposed aggregation mechanism will enhance UDP performance since UDP is connectionless and there are no congestion control mechanisms or retransmissions. Therefore, any asymmetry in bandwidths and/or latency across different paths will impact the performance of UDP applications much less than TCP applications, making UDP the simpler case of the two. We have therefore concentrated most of our efforts on the more diffi cult and interesting case of splitting a TCP connection across multiple IP paths.

As previously mentioned, for TCP, using two or more paths together at the network layer can sometimes lead to worse performance than using a single path alone. This can happen due to the two main mechanisms listed below.

(i) The bandwidth/delay mismatch between paths causes packets sent on a lower capacity path to arrive after the sender-side TCP has already timed out. In this case the late arriving packet needs to be retransmitted. If this were the only drawback one would expect to see only a slight degradation of performance when compared to using a higher capacity path alone. Unfortunately, with each timeout TCP drastically scales back its congestion window and invokes slow-start, thereby under-utilizing the higher capacity path.

(ii) Most TCP implementations include the fast retransmit and fast recovery algorithms [32]. Even if the TCP timeout values were set high enough to prevent the scenario described above, fast retransmit still poses an independent problem. For the purpose of illustration, suppose the ratio of the round-trip times (RTTs) of two paths being used is 4 and suppose the fi rst packet is transmitted on the lower bandwidth path and the next four packets are transmitted on the faster path. The receipt of packets 2,3 , and 4 at the destination will cause the fast retransmission of the fi rst packet (by the receiverside TCP sending three duplicate ACKs for packet 0), thereby wasting the prior transmission on the lower bandwidth path. Worse yet, the recovery phase following a fast retransmission scales back the congestion window. 
Both of these issues are addressed in detail below. We fi rst consider the prevention of timeouts. After a brief overview of the problem in the context of two paths, we present an analytical model for $N$ paths. We then derive expressions that show how much bandwidth discrepancy can be tolerated without incurring timeouts. The issue of fast retransmissions is then examined, with the typical number of duplicate acknowledgments expressed in terms of path bandwidth ratios.

\subsection{TCP Retransmission Timeouts}

For the sake of illustration, consider striping TCP packets across two paths, a high bandwidth and a low bandwidth path. Timeouts will happen only if the RTTs for packets sent across the two paths are substantially different. Noting from (3) that the RTT can be split into two components, namely

$$
\mathrm{RTT}=\frac{p}{b}+\tau,
$$

where $p$ is the packet size, $b$ is the bandwidth of the path, and $\tau$ includes all other delays such as signal propagation delays on all paths, processing delays at all intermediate routers, all delays associated with acknowledgments (ACKs), etc.

If the RTTs for packets sent across the two paths are dominated by their respective $p / b$ ratios, then a bandwidth disparity between the paths causes a corresponding disparity in the RTTs across the paths. This in turn can lead to frequent timeouts degrading performance. On the other hand if the RTT is dominated by $\tau$, then a bandwidth disparity does not lead to a signifi cant difference in RTT. This may be the case if the number of hops is suffi ciently large, or the transmission distance and hence propagation delay is suffi ciently large. In such cases we expect to see performance gains when multiple network interfaces are used simultaneously.

As an example, consider two paths with bandwidths of $160 \mathrm{Kbps}$ and $40 \mathrm{Kbps}$, giving a bandwidth ratio of 4:1. For full Ethernet sized packets (around 1500 bytes per packet) being sent a single hop between adjacent nodes the "other delays" in equation (18) are negligible, since $\tau$ is on the order of microsecond whereas the $p / b$ ratios are 100 s of milliseconds. In this case the RTTs are dominated by the $p / b$ ratios, implying that using the two paths together may be worse than using the higher capacity path by itself. Now consider two paths with the same 4:1 bandwidth ratio, but with actual bandwidths of $100 \mathrm{Mbps}$ and $25 \mathrm{Mbps}$, and where the destination is several hops away. Now the $p / b$ ratio for each path is on the order of 100s of microseconds, whereas $\tau$ can be milliseconds. In this case the RTTs are dominated by $\tau$ implying that using the two paths together can be expected to yield better overall performance than using the higher capacity path alone.

Note that reducing the packet size $p$ will help mitigate the effect of the $p / b$ ratio on the RTT, and hence allow a greater disparity in bandwidths. In fact the packets queued for transmission on the lower capacity path can be dynamically fragmented to equalize the $p / b$ ratios of both paths. Next we present a quantitative analysis of the problem.

In current versions of TCP, the retransmission timeout (RTO) for the $i$-th packet is set as

$$
\mathrm{RTO}_{i}=R_{i-1}+K \cdot V_{i-1},
$$

where $R_{i-1}$ and $V_{i-1}$ are the current smoothed estimates of RTT and the deviation in RTT respectively (just prior to sending the $i$-th packet), and $K$ is a constant factor (typically $K=4$ ). $R_{i}$ and $V_{i}$ are defi ned by the 
following recursive equations.

$$
\begin{gathered}
R_{i}=\alpha \cdot R_{i-1}+(1-\alpha) \mathrm{RTT}_{i} \\
V_{i}=\beta \cdot V_{i-1}+(1-\beta)\left|\mathrm{RTT}_{i}-R_{i}\right|,
\end{gathered}
$$

where $\mathrm{RTT}_{i}$ is the sampled RTT of the $i$-th packet. Equations (20) and (21) act as a low-pass filter on the sampled RTT, which smoothes out variations.

The exact value of RTO will depend on the sequence of RTT samples. In our multiple path scenario, this will depend on the sequence of paths chosen to send packets on. As an approximation, the average RTT and average deviation in RTT will be considered, when packets are sent along paths according to (14).

From relations (7) and (14), the fraction of packets sent along path $i$ is given by

$$
\frac{n_{i}}{n_{\text {total }}}=\frac{b_{i} / p_{i}}{\sum_{k=1}^{N} b_{k} / p_{k}}
$$

Assuming that the RTTs for each path are bandwidth dominated (i.e., $r_{i} \approx p_{i} / b_{i}$ ) this becomes

$$
\frac{n_{i}}{n_{\text {total }}} \approx \frac{1}{r_{i}\left(\sum_{k=1}^{N} 1 / r_{k}\right)}
$$

The average RTT across all paths is then

$$
\bar{r}=\sum_{i=1}^{N} r_{i} \cdot \frac{n_{i}}{n_{\text {total }}}=\frac{N}{\sum_{k=1}^{N} 1 / r_{k}} .
$$

Similarly, the average deviation in RTT is

$$
\bar{v}=\sum_{i=1}^{N}\left|r_{i}-\bar{r}\right| \cdot \frac{n_{i}}{n_{\text {total }}}=\sum_{i=1}^{N} \frac{\left|r_{i}-\bar{r}\right|}{r_{i}\left(\sum_{k=1}^{N} 1 / r_{k}\right)} .
$$

Then from (19), no timeouts will occur if

$$
r_{i}<\bar{r}+K \cdot \bar{v} \quad \text { for } 1 \leq i \leq N
$$

\subsubsection{A Two Path Example}

While (26) ultimately determines if timeouts will occur, a simple two path example is now considered to examine how much of a difference in RTTs can be tolerated before timeouts occur. In this scenario there are two paths between $A$ and $B$, meaning $N=2$. The RTTs of the paths are $r_{1}$ and $r_{2}$ respectively, where we will assume that $r_{1} \leq r_{2}$ and that both are bandwidth dominated. The average RTT is then

$$
\bar{r}=\frac{2 \cdot r_{1} \cdot r_{2}}{r_{1}+r_{2}},
$$

and the average deviation in RTT is

$$
\bar{v}=\frac{2 \cdot r_{1} \cdot r_{2}\left(r_{2}-r_{1}\right)}{\left(r_{1}+r_{2}\right)^{2}} .
$$


Then from (26), no timeout will occur if

$$
r_{i}<\frac{2 \cdot r_{1} \cdot r_{2}}{r_{1}+r_{2}}+\frac{2 \cdot K \cdot r_{1} \cdot r_{2}\left(r_{2}-r_{1}\right)}{\left(r_{1}+r_{2}\right)^{2}} \text { for } r_{i} \in\left\{r_{1}, r_{2}\right\}
$$

From the assumption $r_{1} \leq r_{2}$, it follows that $r_{1} \leq \bar{r}$, and (29) is always satisfi ed for $r_{l}=r_{1}$. Therefore, a timeout can only occur when $r_{i}=r_{2}$.

If the ratio of RTTs is expressed as $\rho$, where

$$
\rho=\frac{r_{2}}{r_{1}} \quad \text { or } \quad r_{1}=\frac{r_{2}}{\rho}
$$

no timeouts will occur if

$$
r_{2}<2 \cdot r_{2}\left[\frac{1}{\rho+1}+\frac{K(\rho-1)}{(\rho+1)^{2}}\right]
$$

This restricts $\rho$ to

$$
1<\rho<2 \cdot K-1 \quad \text { or } \quad 1<\frac{r_{2}}{r_{1}}=\frac{b_{1} \cdot p_{2}}{b_{2} \cdot p_{1}}<2 \cdot K-1
$$

In other words, for two paths using equal packet sizes and the typical value of $K=4$, the path bandwidths can vary by up to a factor of 7 and no timeouts will occur.

When packet sizes are not equal, (32) places the following upper bound on the ratio of path bandwidths, denoted $\phi$ :

$$
\phi=\frac{b_{1}}{b_{2}}<\frac{p_{1}}{p_{2}}(2 \cdot K-1) .
$$

The signifi cance of constraint (33) is that the ratio of packet sizes effectively scales the timeout threshold, which then allows a greater bandwidth disparity to be tolerated. For example, consider using a $1 \mathrm{Mbps}$ wireless LAN connection along with a $40 \mathrm{Kbps}$ cellular phone modem. In this case the ratio of bandwidths of the two paths is 25 . Therefore, to avoid time-outs, the packet size ratio must be set greater than (25/7):1 as dictated by constraint (33).

We would like to point out that in many cases it is possible to appropriately size packets sent along different paths in a manner completely transparent to TCP by fragmenting at the IP layer. Assuming suffi ciently large packet sizes, each packet which TCP sends down to IP could be fragmented into the appropriate number of pieces, each of correct size. These fragments could then be distributed across paths in the right proportions so that constraint (33) is satisfi ed. This fragmenting is, however, slightly more involved than the normal IP fragmentation needed when forwarding between networks with different MTUs and interferes with the path MTU discovery mechanism used by TCP.

Note that the restrictions stipulated in (33) apply only if the paths are bandwidth dominated, i.e. if $p / b \gg \tau$ in (18). Propagation and queuing delays at intermediate routers as well as the ACK reception time all contribute to $\tau$. If all packet sizes, including the largest packet size which will be sent on the highest bandwidth path, are such that $p / b<\tau$ then the paths are no longer bandwidth dominated. In this case, RTTs are dominated by $\tau$ which is probably similar for all paths. In other words, the RTTs are approximately equalized thereby making it possible to stripe the connection across all paths without incurring timeouts, irrespective of the bandwidth ratios involved. 


\subsubsection{The Effect Smoothed Values Have on RTO}

Note that use of the exact mean values $\bar{r}, \bar{v}$ in (26) is an idealized approximation. In reality the smoothed estimates for RTT and deviation in RTT from (20) and (21) play an substantial role in determining what difference in RTTs will cause timeouts. We extracted the TCP RTO timer code from the FreeBSD kernel to evaluate the effect of this smoothing.

A sequence of 100,000 RTT values was given as input to the RTO timer code. This sequence was generated by assuming two paths with bandwidths $b_{1}$ and $b_{2}$. Packets were probabilistically striped across the two paths, where the probabilities corresponded to bandwidth fractions in accordance with (22). Packets sent on the fi rst and second paths were assumed to have sizes $p_{1}$ and $p_{2}$ respectively. The RTT value for a packet was then determined based on its size and the bandwidth of the path it was sent on. If the RTT exceeded the smoothed RTO from the RTO timer code, defi ned in (19), it was considered a timeout.

For equal sized packets, the fraction of packets sent on the lower bandwidth path that lead to timeouts as a function of path bandwidth ratio, $\phi$, is show in Figure 2(a). The curve labeled "Ideal" in the fi gure corresponds to ideal behavior as predicted by (33). That is, no timeouts occur for bandwidth ratios of $\phi<7$ and timeouts occur for $100 \%$ of the packets for $\phi \geq 7$, resulting in a step-function. The curve labeled "Actual" shows the results from the RTO timer for $K=4$ and the default values of $\alpha=7 / 8$ and $\beta=3 / 4$ for the smoothing coeffi cients from (20) and (21). The plot shows that using smoothed RTT values for the RTO estimation causes more timeouts than predicted by the ideal curve. This demonstrates that smoothing has a signifi cant impact on the number of timeouts. To further clarify the impact of smoothing we have also included the curve labeled " $\alpha=0.99 \beta=0.99$ " which approximates the idealized step-function much better than the default values. This curve is included only to confi rm the analytical results, where by having such high smoothing coeffi cients the long-term averages are tracked more closely and the influence of short-term transient fluctuations is diminished.

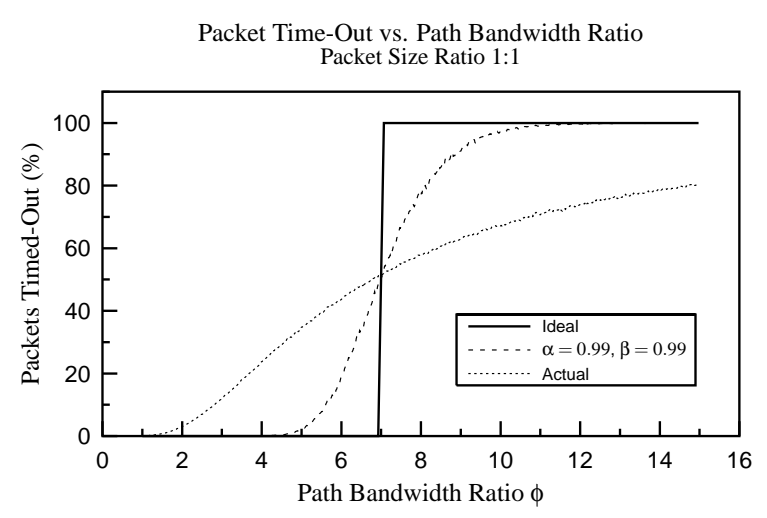

(a) Packet Size Ratio 1:1

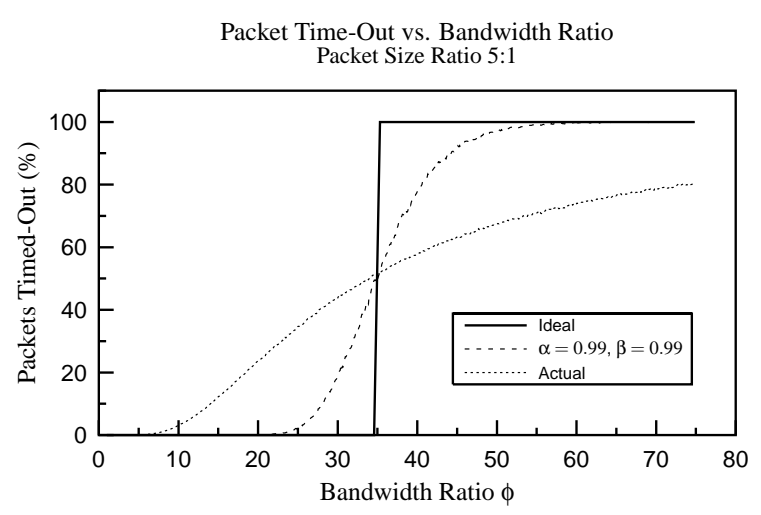

(b) Packet Size Ratio 5:1

Figure 2: Lower Bandwidth Path Packet Timeout Percentage

As previously mentioned, packet sizes can be adjusted to equalize RTTs. To test this hypothesis we conducted the same set of experiments for a packet size ratio of 5:1, with the results shown in Figure 2(b). Note that both experimental plots in Figure 2(b) indicate that the bandwidth ratio threshold has been scaled 
by the packet size ratio of $5: 1$, thereby confi rming (33).

\subsubsection{Periodic Smoothed RTT and RTO Values}

As Figure 2 illustrates, the use of smoothed RTT and deviation in RTT values in the retransmission timeout calculation leads to a signifi cant discrepancy between the predicted and actual bandwidth ratios that avoid timeouts. When packets are striped nondeterministically according to a given probability distribution, the empirical limit on the maximum tolerable bandwidth ratio is much less than that predicted by (33). In order to see how this might affect an actual packet striping implementation we now consider the special case when sampled RTT values form a repeating periodic sequence. This can occur when packets are striped among available paths by a deterministic scheduling algorithm which distributes data in approximate accordance with (14), for example Defi cit or Surplus Round-Robin (DRR, SRR) [33, 34].

In such a scenario, suppose the sampled RTT values repeat every $T$ packets, that is for the $i$-th sampled RTT value $r_{i}, r_{i+T}=r_{i}$. This in turn makes the steady-state smoothed RTT values repeat with period of $T$, or $R_{i+T}=R_{i}$. In this case exact expressions for the steady-state smoothed values of RTT and deviation in RTT can be found as follows.

From (20), a sequence of $T$ sampled RTT values $r_{1}, r_{2}, \cdots, r_{T}$ leads to the system of equations given below.

$$
\begin{aligned}
& R_{1}=\alpha \cdot R_{0}+(1-\alpha) r_{1} \quad R_{1}-\alpha \cdot R_{0}=(1-\alpha) r_{1} \\
& \vdots \quad \text { or } \\
& R_{T}=\alpha \cdot R_{T-1}+(1-\alpha) r_{T} \quad R_{T}-\alpha \cdot R_{T-1}=(1-\alpha) r_{T}
\end{aligned}
$$

Noting that $R_{0}=R_{T}$, this system or equations can be expressed in matrix form as

$$
\mathbf{A R}=\mathbf{r},
$$

where $\mathbf{R}$ corresponds to the smoothed RTT values, $\mathbf{r}$ is the sampled RTT sequence, and $\mathbf{A}$ is a $T \times T$ matrix determined by

$$
\mathbf{A}_{i j}= \begin{cases}1 & \text { if } j=i \\ -\alpha & \text { if } j=(i-2 \bmod T)+1 \\ 0 & \text { otherwise }\end{cases}
$$

Expression (35) then becomes

$$
\underbrace{\left[\begin{array}{ccccc}
1 & & & & -\alpha \\
-\alpha & 1 & & & \\
& -\alpha & 1 & & \\
& & \ddots & \ddots & \\
& & & -\alpha & 1
\end{array}\right]}_{\mathbf{A}} \underbrace{\left[\begin{array}{c}
R_{1} \\
R_{2} \\
R_{3} \\
\vdots \\
R_{T}
\end{array}\right]}_{\mathbf{R}}=(1-\alpha) \underbrace{\left[\begin{array}{c}
r_{1} \\
r_{2} \\
r_{3} \\
\vdots \\
r_{T}
\end{array}\right]}_{\mathbf{r}} .
$$

Since $\mathbf{A}$ is nonsingular, the steady-state smoothed RTT values can be found as

$$
\mathbf{R}=(1-\alpha) \mathbf{A}^{-1} \mathbf{r} .
$$


The structure of A leads to the following solution for the smoothed RTT values given by (38).

$$
R_{i}=\frac{1-\alpha}{1-\alpha^{T}} \sum_{k=1}^{T} r_{k} \cdot \alpha^{(i-k \bmod T)} .
$$

The steady-state smoothed deviation in RTT values, from (21), can be expressed in a similar manner as

$$
V_{i}=\frac{1-\beta}{1-\beta^{T}} \sum_{k=1}^{T}\left|r_{k}-R_{k}\right| \cdot \beta^{(i-k \bmod T)} .
$$

Therefore from (19), (39), and (40), a periodic sequence of sampled RTT values will not result in any steady-state timeouts if

$$
r_{i}<R_{i-1 \bmod T}+K \cdot V_{i-1 \bmod T} \text { for } \quad 1 \leq i \leq T \text {. }
$$

\subsubsection{A Periodic Two Path Example}

From (41) the exact distribution of timeouts can be effi ciently found for any periodic sequence of RTTs. To see how this actually manifests itself, an example two path scenario is considered analogous to that of Section 4.1.1. In this case packets are split between two paths in a deterministic periodic fashion. Assume $T-1$ consecutive packets are sent on the first path having a RTT of $r_{1}$ followed by a single packet sent on the second path with RTT $r_{2}$, where $r_{1} \leq r_{2}$. In other words, the fi rst path is assumed to be of higher bandwidth than the second, and the packets sent generate a periodic pattern of sampled RTT values which repeats every $T$ packets. In this periodic two path case a retransmission timeout will occur only for a packet sent on the lower bandwidth path when $r_{2}>\mathrm{RTO}_{T}$. From (19), the condition necessary to avoid timeouts is then

$$
r_{2}<R_{T-1}+K \cdot V_{T-1}
$$

which can be determined explicitly from (39) and (40).

For this periodic two path case, the $i$-th smoothed RTT from (39) can be simplifi ed and expressed as

$$
R_{i}=r_{1}+\frac{\alpha^{i \bmod T}(1-\alpha)\left(r_{2}-r_{1}\right)}{1-\alpha^{T}} .
$$

The smoothed RTT just prior to when the $T$-th packet is sent on the lower bandwidth path is then

$$
R_{T-1}=\frac{\left(1-\alpha^{T-1}\right) r_{1}+\left(\alpha^{T-1}-\alpha^{T}\right) r_{2}}{1-\alpha^{T}} .
$$

The smoothed deviation in RTT needed for (42), $V_{T-1}$, can then be found from (40) and (43), and by noting that the smoothed RTT is bounded by $r_{1} \leq R_{i} \leq r_{2}$. This becomes

$$
V_{T-1}=\frac{(1-\beta)\left(r_{2}-r_{1}\right)}{\left(1-\beta^{T}\right)\left(1-\alpha^{T}\right)}\left[\frac{(1-\alpha)\left(\alpha^{T}-\alpha \beta^{T-1}\right)}{\alpha-\beta}+\beta^{T-1}\left(\alpha-\alpha^{T}\right)\right]
$$

To ensure that the fraction of data sent on each path is consistent with (14), the period $T$ must depend on both the packet sizes used for each path and the path bandwidths. From (14), the fraction of data sent on each path equals the ratio of path bandwidth to total bandwidth, or if $d_{i}$ is the amount of data sent on path $i$ :

$$
\frac{d_{1}}{d_{1}+d_{2}}=\frac{b_{1}}{b_{1}+b_{2}} \quad \text { or } \quad \frac{n_{1} \cdot p_{1}}{n_{1} \cdot p_{1}+n_{2} \cdot p_{2}}=\frac{p_{1} / r_{1}}{p_{1} / r_{1}+p_{2} / r_{2}}
$$


where $b_{i}, n_{i}$, and $p_{i}$ are respectively the bandwidth, number of packets, and packet size for path $i$. This results in

$$
\frac{n_{1}}{n_{2}}=\frac{r_{2}}{r_{1}}
$$

Therefore, for every one packet sent on the lower bandwidth path there are $n_{1} / n_{2}$ packets sent on the higher bandwidth path, or by letting $\rho=r_{2} / r_{1}$ the period becomes

$$
T=\rho+1
$$

From (44), (45), and (48) the retransmission timeout condition necessary to avoid timeouts, from (42), can be expressed equivalently as

$$
(\rho-1)\left(1-\frac{K \alpha(1-\beta)}{1-\beta^{\rho+1}}\left[\frac{(1-\alpha)\left(\alpha^{\rho}-\beta^{\rho}\right)}{\left(1-\alpha^{\rho}\right)(\alpha-\beta)}+\beta^{\rho}\right]\right)<0 .
$$

This can be solved numerically for the default values of $\alpha=7 / 8, \beta=3 / 4$, and $K=4$ which, for the range of interest $(\rho \geq 1)$, gives

$$
1<\rho<4.386 \cdots \quad \text { or } \quad 1<\frac{b_{1} \cdot p_{2}}{b_{2} \cdot p_{1}}<4.386 \cdots .
$$

Expressed in terms of path bandwidth ratio $\phi$, the upper bound becomes

$$
\phi<\frac{p_{1}}{p_{2}} 4.386 \cdots
$$

In other words for this simple periodic case, smoothed RTT and deviation in RTT values reduce the tolerable path bandwidth disparity from the factor of 7 predicted by (32) to approximately a factor of 4 . (When equal sizes packets are sent on both paths and typical values are used for the TCP constants.)

This is verifi ed experimentally in the manner described in Section 4.1.2, with the results shown in Figure 3. In this case packets are split deterministically between the two paths using the SRR scheduling algorithm [34]. As in Figure 2, the tolerable bandwidth ratio is scaled by the ratio of packet sizes used. However, due to the deterministic nature of the sampled RTT values, there is a strict maximum bandwidth ratio which will not cause spontaneous retransmission timeouts in steady-state. Note that in some cases the SRR scheduling algorithm gives rise to a more complicated periodic sequence than the $T-1$ packets followed by 1 packet used in the analytical model, for example when the ratio of path RTTs, $\rho$, is non-integer. For this reason, the data plotted in Figure 3 differs slightly from what (51) predicts.

\subsection{TCP Fast Retransmissions}

As mentioned at the beginning of this section, the second mechanism that can enable a lower bandwidth path to drag down a higher bandwidth path is the fast retransmission and recovery algorithms found in almost every TCP implementation today. These algorithms are independent of the regular timeout mechanisms.

A TCP sender using the fast retransmit algorithm infers that a packet was lost after receiving a suffi cient number of duplicate ACKs, typically 3. Since a lost packet implies congestion, congestion avoidance procedures are then invoked. This mechanism can be falsely triggered, i.e. when no packet loss has occurred, 


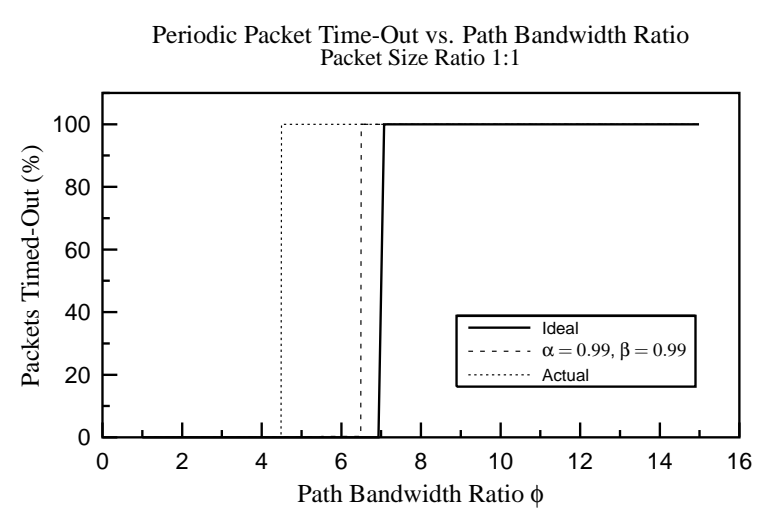

(a) Packet Size Ratio 1:1

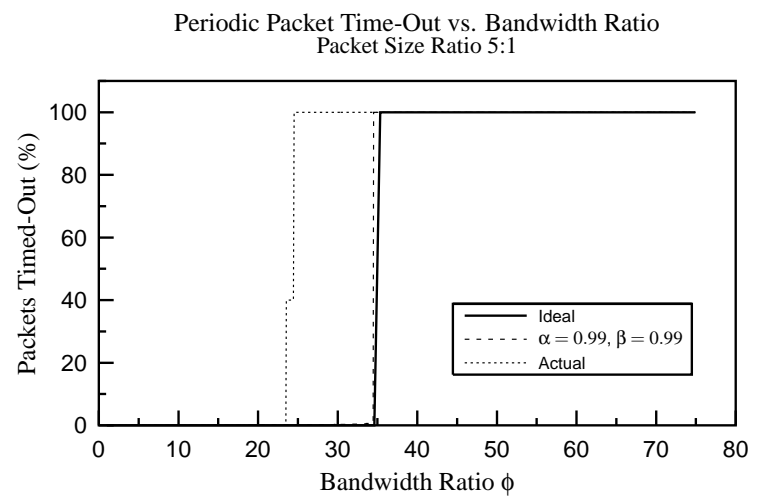

(b) Packet Size Ratio 5:1

Figure 3: Periodic Lower Bandwidth Path Packet Timeout Percentage

when out-of-order packets are received the destination. This can happen frequently in our bandwidth agregation scheme, since multiple packets may be received from higher bandwidth paths while a packet sent on a lower bandwidth path is in flight. Note that even when delayed ACKs are used, a TCP receiver will immediately generate an ACK for each out-of-order packet it receives.

The maximum number of legitimate duplicate ACKs (not caused by packet loss) that a TCP sender using our bandwidth agregation scheme can expect is characterized as follows. Assuming a TCP receiver processing packets in the order of reception, the number of duplicate ACKs generated will equal the number of packets received on all other paths while a single packet is in flight on the path with the largest one-way latency. More specifi cally, let $k$ be the one-way latency of path $k$, which is greater than or equal to the one-way latency of any other path, or

$$
l_{k}=\max \left(\frac{p_{1}}{b_{1}}, \frac{p_{2}}{b_{2}}, \cdots, \frac{p_{N}}{b_{N}}\right) .
$$

The maximum number of duplicate ACKs are then generated when packets are continually streaming in from all other paths. Noting that the number of packets received from path $i$ in time $l_{k}$ is $l_{k} \cdot p_{i} / b_{i}$, the number of duplicate ACKs in this case, DupACKs, is then

$$
\text { DupACKs }=\sum_{i=1}^{N}\left\lfloor l_{k} \frac{b_{i}}{p_{i}}\right\rfloor-1 \leq l_{k} \sum_{i=1}^{N} \frac{b_{i}}{p_{i}}-1 .
$$

When equal sized packets are used for all paths, this upper bound becomes

$$
\text { DupACKs } \leq \frac{1}{b_{k}} \sum_{i=1}^{N} b_{i}-1,
$$

where $b_{k}$ is the minimum path bandwidth. In the two path scenario the right side of (54) is simply the ratio of path bandwidths, or DupACKs $\leq \phi$.

The impact that the fast retransmit and recovery algorithms have on TCP performance when persistent packet reordering occurs is illustrated in Figure 4. This plot presents simulation data generated by the ns- 2 
network simulator [35]. The two path scenario was considered, where the total raw bandwidth between the two communicating nodes was $20 \mathrm{Mbps}$, and bandwidth ratio of the two paths was varied from $1 \leq \phi \leq 15$ in integral steps. Equal sized packets of 1000 bytes were split deterministically between the paths using the SRR algorithm, conforming to (14). Each data point shown is the average resulting from 10 runs, each lasting 4 simulated minutes The portion of total raw bandwidth realized by TCP is shown, as well as the number of fast retransmissions, as a function of bandwidth ratio. The number of fast retransmissions is represented as a percentage found by dividing the number of fast retransmissions that occurred by the number of packets sent on the lower bandwidth path. Clearly TCP experiences a signifi cantly performance degradation corresponding to the sudden increase in fast retransmissions for bandwidth ratios of $\phi \geq 3$ (due to duplicate ACKs).

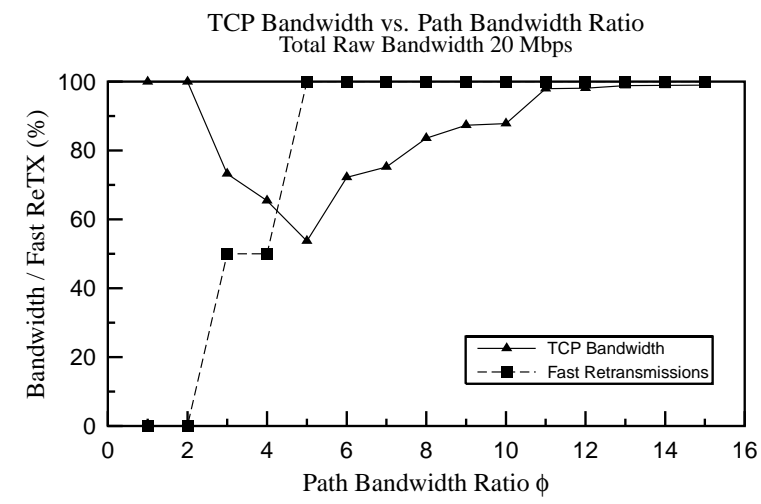

Figure 4: Simulated TCP Throughput for Fixed Raw Bandwidth

Fast retransmissions can be avoided by out-of-order transmission. For instance, assume that there are two paths between the source and destination with a bandwidth ratio $\phi=4$. As per equation (14), the optimal load distribution in this case is 1 packet on the lower bandwidth path for every 4 packets transmitted on the higher bandwidth path. If packet 1 is transmitted fi rst on the lower bandwidth path followed by packets 2,3,4,5 on the higher bandwidth path, the reception of packet 4 will cause a fast retransmission of packet 1. The solution to this problem is to send packet number 5 on the lower bandwidth path first and then transmit packets 1,2,3,4 on the higher bandwidth path. Assuming that TCP pushes enough segments down to the network layer, the proposed out-of-order transmission can be done transparently by the network layer, however the network layer must then understand TCP segment numbers.

\subsection{TCP Performance Summary}

We conclude this section by summarizing ways of tuning the behavior of TCP. Based on the above discussion it is seen that TCP can be optimized in several ways, including the following.

(i) Set large window sizes to accommodate all the packets when using paths with high bandwidth ratios.

(ii) Reduce the packet size $p$ appropriately for each path to reduce the bandwidth domination of RTTs.

(iii) Set RTO values high by either allowing a greater variation in RTT or by confi guring somewhat high absolute minimum values. As shown above, the default value of $K$ allows for a bandwidth ratio of 7 
when equal sized packets are used. A value of $K=13$ covers paths with bandwidth ratios up to 25 .

(iv) Use TCP Vegas and other enhanced TCP versions that mitigate the effect of slow-start.

(v) Allow sending packets out-of-order to avoid fast retransmissions.

Note that all the above modifi cations and optimizations can be confi ned to the sender side alone. They preserve the end-to-end TCP semantics and will transparently inter-operate with any standard TCP receiver.

\section{Implementation and Results}

We have tested a proof-of-concept implementation where the sender and receiver were connected by two logical paths. We implemented the above mentioned tunneling mechanism on a FreeBSD sender and receiver. The dummynet facility [36] was used in our experiments. This allows the kernel to route packets through logical paths based on specifi ed probabilities.

For our experiments, we created two logical paths over a single physical path. We would like to emphasize that it would be just as easy to run the experiment across two different physical paths. The logical implementation we chose offered a greater flexibility in controlling both the bandwidth of each path and the probabilities with which each path was chosen, which in turn determined packet load distribution.

The fi rewalling mechanisms provided by FreeBSD were exploited on the sending side to set the proper source address for each packet and to encapsulate packets going through one of the paths. In this case equal sized 1500 byte packets were split probabilistically between the two paths. Similarly, the receiving side was confi gured to strip encapsulating headers as needed. Further details regarding the experimental setup have been omitted for the sake of brevity.

The experimental results are illustrated in Figure 5(a) and Figure 5(b). These Figures show TCP bandwidth as a function of the raw bandwidth, which refers to the total bandwidth of both paths between the source and the destination. TCP bandwidth was measured using the netperf benchmarking package [37].

Figure 5(a) shows the case when both paths have equal bandwidths. The three curves illustrate the bandwidth TCP achieves as a percentage of the total raw bandwidth available when only one path is used, when both paths are used without tunneling, and when both paths are used with tunneling. As expected, over several orders of magnitude, TCP realizes roughly twice the bandwidth when both paths are used simultaneously compared to when only a single path is used. The similarity of the with and without tunneling curves shows that the impact of tunneling on TCP performance is negligible. Since a standard TCP connection is equivalent to using only a single path, this demonstrates that the proposed bandwidth aggregation mechanism can, in principle, deliver additional bandwidth to the application level.

To test the effi cacy of the proposed mechanism we carried out experiments on two paths having different bandwidths. As per the previous analysis for bandwidth dominated paths that have disparate bandwidths, the default value of $K=4$ in most TCP implementations will ideally allow connection striping across paths with bandwidth ratio of $\phi<7$ without incurring timeouts. For our experiments we selected a bandwidth ratio of $\phi=3$ in order to have a reasonable difference in path bandwidths while at the same time pushing the upper limit of the default tolerance for duplicate ACKs before fast retransmissions might occur. The results are illustrated in Figure 5(b), with the curves being analogous to those in Figure 5(a). 


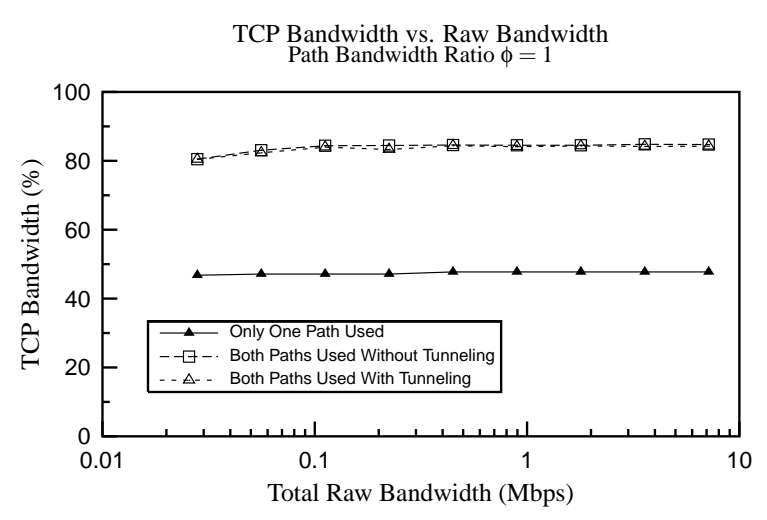

(a) Equal Bandwidth Paths

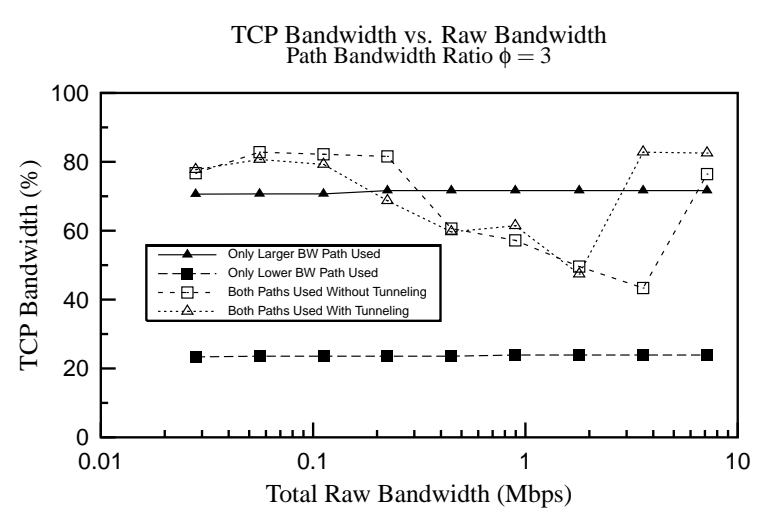

(b) Bandwidth Ratio $\phi=3$

Figure 5: TCP Throughput for Fixed Bandwidth Ratios

The data shows that despite a 3:1 bandwidth disparity, simultaneous use of both paths can yield better performance. For data points at low raw bandwidth values, about $3 \mathrm{Mbps}$ and below, some timeouts do occur which is consistent with the data from Figure 2(a). This result is due to the probabilistic splitting and the effect of using smoothed RTTs as previously explained. The timeouts do cause the lower bandwidth path to drag down the higher bandwidth path somewhat.

For raw bandwidths above about $3 \mathrm{Mbps}$, the RTTs of both paths drop to suffi ciently low values, which in turn causes the RTO values to drop below 1 second. The version of the FreeBSD kernel used for these experiments imposed a lower limit of 1 second for RTO, below which timeouts will not occur. Note that the paths are still bandwidth dominated, i.e. the RTT values are still dominated by the bandwidth, but the timeout values are now suffi ciently large to accommodate the variation in RTT, which prevents timeouts. The net result is that the connection striping across two paths provides higher bandwidth than using the higher bandwidth path by itself, demonstrating that the proposed mechanism works even for bandwidth dominated paths, as long as TCP timeouts can be mitigated.

To further investigate the limitations of our bandwidth aggregation scheme, TCP performance was investigated over a range of bandwidth ratios while the total raw bandwidth available was held constant. These experiments were again performed with two network paths between communicating hosts, however tunneling was omitted since its impact is negligible. This time equal sized 1500 byte packets were split deterministically between the two paths. The results are shown in Figure 6(a) and Figure 6(b). Both plots show the TCP bandwidth achieved when both paths are used, and when only the higher bandwidth path is used alone. These results agree closely with (51) and Figure 3(a), showing that a bandwidth ratio of around 4 can be tolerated when deterministic packet splitting is employed. 


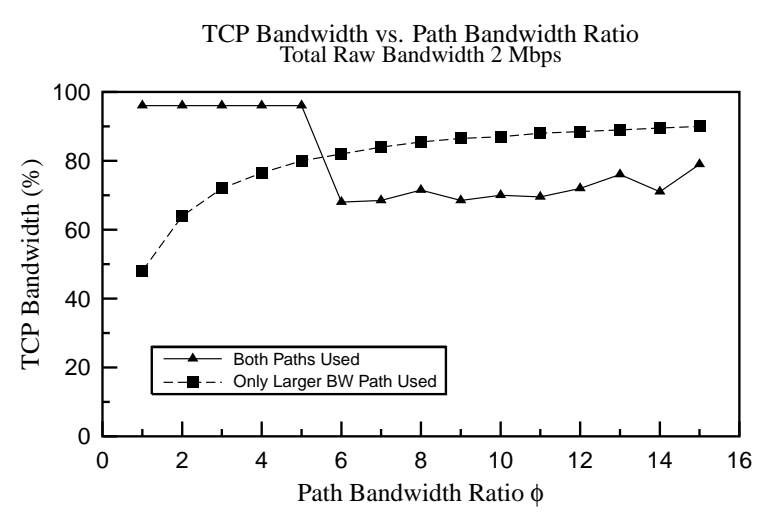

(a) 2 Mbps Total Raw Bandwidth

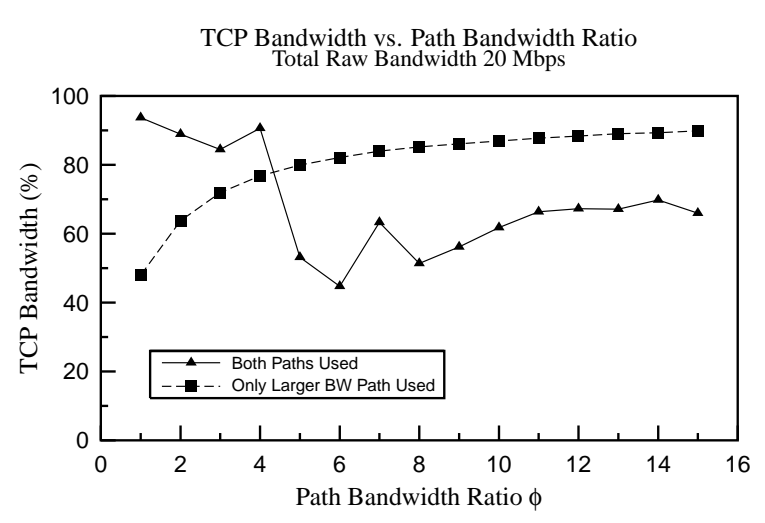

(b) 20 Mbps Total Raw Bandwidth

Figure 6: TCP Throughput for Fixed Raw Bandwidth

\section{Conclusions}

We have proposed a novel mechanism to stream data simultaneously across multiple IP paths in order to aggregate their bandwidth. It is applicable to connectionless (UDP) flows as well as for striping the data flow in a TCP connection across multiple IP paths. We investigated its performance and experimentally validated the analytical results. The work was motivated by practical considerations. As ubiquitous network access is becoming a reality the number of wireless data transmission technologies continues to grow. It is therefore clear that in the near future, there will be multiple transport conduits. Our analysis and data demonstrate that the proposed mechanism works well in most such scenarios of practical interest.

Future work includes an implementation of all the TCP modifi cations and optimizations mentioned above and more detailed simulations in wireless and mobile scenarios. Another approach is to implement the load sharing capability in SCTP and compare it with the mechanism proposed herein. Lastly, dynamically adding or deleting IP addresses from a connection involving end-points with multiple interfaces could lead to security hazards which warrant further investigation.

\section{References}

[1] G. Malkin, Nortel Networks Multi-link Multi-node PPP Bundle Discovery Protocol, September 1999. RFC 2701.

[2] K. Sklower, B. Lloyd, G. McGregor, D. Carr, and T. Coradetti, The PPP Multilink Protocol (MP), August 1996. RFC 1990.

[3] K. Sklower, B. Lloyd, G. McGregor, and D. Carr, The PPP Multilink Protocol (MP), November 1994. RFC 1717.

[4] E. W. Simpson, The Point-to-Point Protocol (PPP), July 1994. RFC 1661.

[5] E. W. Simpson, PPP in HDLC-like Framing, July 1994. RFC 1662. 
[6] D. Rand, PPP Reliable Transmission, July 1994. RFC 1663.

[7] R. Ogier, V. Ruenburg, and N. Shacham, "Distributed algorithms for computing shortest pairs of disjoint paths," IEEE Transactions on Information Theory, vol. 39, pp. 443-455, Mar. 1993.

[8] I. Cidon, R. Rom, and Y. Shavim, "Analysis of multi-path routing," IEEE/ACM Transactions on Networking, vol. 7, pp. 885-896, Dec. 1999.

[9] N. Taft-Plotkin, B. Bellur, and R. Ogier, "Quality of Service Routing Using Maximally Disjoint Paths," in Proceedings of the IEEE IWQoS'99, London, UK., pp. 119-128, June 1999.

[10] A. Nasipuri and S. R. Das, "On-Demand Multipath Routing for Ad-Hoc Networks," in Proceedings of the IEEE ICCCN,99, Boston, pp. 64-70, October 1999.

[11] J. Raju and J. Garcia-Luna-Aceves, "A New Approach to On-Demand Multipath Routing," in Proceedings of the IEEE ICCCN,99, Boston, pp. 522-527, October 1999.

[12] M. Pearlman, Z. Haas, P. Scholander, and S. Tabrizi, "On the Impact of Alternate Path Routing for Load Balancing in Mobile Ad Hoc Networks," in Proceedings of ACM MobiHOC'00, Boston, pp. 119-128, August 2000.

[13] M. G. Sung-Ju Lee, "Split Multipath Routing with Maximally Disjoint Paths in Ad hoc Networks," in Proceedings of ICC'01, Helsinki, Finland, June 2001.

[14] W-H Liao et. al., "A Multi-Path QoS Routing Protocol in a Wireless Mobile Ad Hoc Network," in Proceedings of the IEEE ICN'OO, CREF, Colmar, France, July 2001.

[15] A. Snoeren, “Adaptive Inverse Multiplexing for Wide-Area Wirelss Networks," in IEEE Globecom, pp. 1665-1672, 1999.

[16] H. Adiseshu and G. Parulkar and G. Varghese, "A Reliable and Scalable Striping Protocol," in ACM SIGCOMM, pp. 131-141, 1996.

[17] D. C. Anderson, J. S. Chase, and A. M. Vahdat, "Interposed request routing for scalable network storage," in Proceedings of the Fourth Symposium on Operating System Design and Implementation (OSDI), October 2000.

[18] Sun Microsystems, "Sun StorEdge Network Data Replicator White Paper." http://www. sun.com/storage/white-papers/sndr.html.

[19] A. Watson, Network Appliance, Inc., "Filer Deployment Strategies for Evolving LAN Topologies." http://www.netapp.com/tech_library/3009.html.

[20] R. Haagens, "iSCSI requirements." http://www.ietf.org/proceedings/00jul/SLIDES/ips-iscsi-reqs.pdf.

[21] Cisco Systems, "Etherchannel technologies." http://www.cisco.com/warp/public/779/largeent/learn/technologies/fast_echannel.html.

[22] "SCTP Reference Implementation." http://www. sctp.org/.

[23] “GPL SCTP Prototype Implementation.” www.sctp.de/.

[24] "SCTP for beginners." http://tdrwww.exp-math.uni-essen.de/pages/forschung/sctp_fb.

[25] "SCTP: An Overview." http://sctp.chicago.il.us/sctpoverview.html.

[26] "Protocol Engineering Lab at University of Delaware CIS Dept." http://www.cis.udel.edu/〜iyengar/research/SCTP. 
[27] R. R. Stewart, M. A. Ramalho et. al., "SCTP Extensions for Dynamic Reconfi guration of IP Addresses and Enforcement of Flow and Message Limits," June 2001. http://www.ietf.org/internet-drafts/draft-ietf-tsvwg-addip-sctp-02.txt.

[28] E. C. Perkins, IP Mobility Support, October 1996. RFC 2002.

[29] C. Perkins, IP Encapsulation within IP, October 1996. RFC 2003.

[30] C. Perkins, Minimal Encapsulation within IP, October 1996. RFC 2004.

[31] J. Solomon, Applicability Statement for IP Mobility Support, October 1996. RFC 2005.

[32] V. Jacobson, "Modifi ed TCP Congestion Avoidance Algorithm." end2end interest group mailing list, April 1990.

[33] M. Shreedhar and G. Varghese, "Effi cient fair queuing using defi cit round robin," in SIGCOMM: ACM Special Interest Group on Data Communication, (Cambridge, MA), pp. 231-242, September 1995.

[34] H. Adiseshu, G. Parulkar, and G. Varghese, "A reliable and scalable striping protocol," in SIGCOMM: ACM Special Interest Group on Data Communication, (Palo Alto, CA), pp. 131-141, August 1996.

[35] S. McCanne and S. Floyd, "ns network simulator." http://www.isi.edu/nsnam/ns/.

[36] L. Rizzo, "Dummynet: a simple approach to the evaluation of network protocols," ACM Computer Communication Review, vol. 27, pp. 31-41, Jan. 1997.

[37] Information Networks Division, Hewlett-Packard Company, Netperf: A Network Performance Benchmark, February 15 1996. http://www. netperf.org/. 